\title{
Undulator Based Synchrotron Radiation Source in the 5-30 eV Spectral Region *
}

\author{
Xiaohao Zhang, James B. Murphy and Samuel Kripsky \\ National Synchrotron Light Source \\ Brookhaven National Laboratory \\ Upton, NY 11973

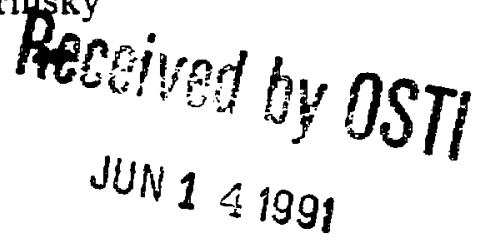

\begin{abstract}
There has been recent interest in the utilization of high intensity radiation in the $5-30 \mathrm{eV}$ spectral region. Applications include angle resclved photoemission, photochemistry, and radiation oncology. Here we present the design of a $500 \mathrm{MeV}$ electron storage ring with long insertions for undularors. The ring is designed to provide an emittance of $0.1 \mathrm{~mm}-\mathrm{mrad}$, an average current of $1 \mathrm{Amp}$ and an electron beam lifetime of greater than 3 hrs. The undulators operating near $\mathrm{K}=1$, yield $10^{15} \mathrm{ph} / \mathrm{sec} / 0.1 \%$ bandwidth in the fundamental.
\end{abstract}

\section{Introduction}

Existing second-generation and planned third-generation synchrotron radiation facilities do not provide optimum undulator sources of soft photons in the energy range 5$30 \mathrm{eV}$. In this note we discuss the design of a low energy storage ring with long dispersion free straight sections for soft photon undulators. The electron energy is chosen to be $500 \mathrm{MeV}$. Lower energy leads to shorter undulator period length and hence higher brightness, but, at the cost of reduced tunability of the undulator radiation wavelength. We have taken a relatively large value of the electron emittance, $0.1 \mathrm{~mm}$-mrad. Reduced emittance leads to higher brightness, but at the cost of a shorter Touschek lifetime. The storage ring is designed with 3 superperiods of an expanded Chasman-Green design. With this choice the emittance of $0.1 \mathrm{~mm}$-mrad is achieved with a simple, natural optics. With 4 superperiods, the ring tends to have smaller emittance requiring a more difficult optics to raise the emittance while maintaining the proper $\beta$-function structure for efficient chromaticity correction. In addition the 3 superperiod ring is smaller and more economical.

-This work was performed under the auspices of the U.S. Department of Energy.

\section{Undulators}

The fundamental photon energy produced by an undulator is given by the electron beam energy $E$, the period $\lambda_{4}$ and the magnetic parameter $\mathrm{K}\left(K=.934 B_{u}[T] \lambda_{u}[\mathrm{~cm}]\right)$ of the undulator $[1,2]$. For permanent-magnet undulators, the magnetic field $B_{u}$ is determined by the choice of material and the undulator gap. The tunability of the radiated photon energy is obtained by varying either the electron energy or the gap of the undulator.

Since the required spectrum $(5-30 \mathrm{eV})$ is too wide to be spanned in the first harmonic by a single undulator, we use two undulators with different periods to cover the desired spectral region. Even so, the $\mathrm{K}$ value for high photon energy is still low. In fact, at this case we can increase electron energy to yield higher photon flux. For the 500 $\mathrm{MeV}$ electron storage ring, the periods of undulators are chosen to be $\lambda_{u}=7.5 \mathrm{~cm}$ and $\lambda_{u}=10 \mathrm{~cm}$ respectively. The magnetic parameters $\mathrm{K}$ and the gap of the undulators are plotted in Fig. 1 as functions of the fundamental pho ton energy $\epsilon_{1}$. The main parameters of the undulators are listed in Table 1.

Table 1: Undulator Parameters $\left(\mathrm{SmCo}_{5}\right)$

\begin{tabular}{|l|c|c|}
\hline$\lambda_{u}[\mathrm{~cm}]$ & 7.5 & 10 \\
\hline Photon Energy [eV] & $15 \sim 30$ & $5 \sim 15$ \\
\hline$N_{u}$ & 100 & 75 \\
\hline$L_{u}[\mathrm{~m}]$ & 7.5 & 7.5 \\
\hline $\mathrm{K}$ & $.331 \sim 1.49$ & $1.08 \sim 2.738$ \\
\hline$B_{u}[\mathrm{~T}]$ & $.047 \sim .213$ & $.116 \sim 293$ \\
\hline Full Gap [cm] & $4.7 \sim 8.2$ & $5.3 \sim 8.2$ \\
\hline
\end{tabular}

Since the photon energy of the undulator radiation is related to the magnetic parameter $K$, the number of photons at different energy varies due to the change of the gap. To make sure that there is a enough photon flux, we need to estimate the values at $\epsilon_{1}=30$ at which. the photon flux is a minimum for the fundamental. Using the parameters of the case $30 \mathrm{eV}$ given above, we find flux integrated over 


\section{DISCLAIMER}

This report was prepared as an account of work sponsored by an agency of the United States Government. Neither the United States Government nor any agency thereof, nor any of their employees, makes any warranty, express or implied, or assumes any legal liability or responsibility for the accuracy, completeness, or usefulness of any information, apparatus, product, or process disclosed, or represents that its use wculd not infringe privately owned rights. Reference herein to any specific commercial product, process, or service by trade name, trademark, manufacturer, or otherwise does not necessarily constitute or imply its endorsement, recom. mendation, or favoring by the United States Government or any agency thereaf. The views and opinions of authors expressed herein do not necessarily state or reflect those of the United States Government or any agency thereor. 

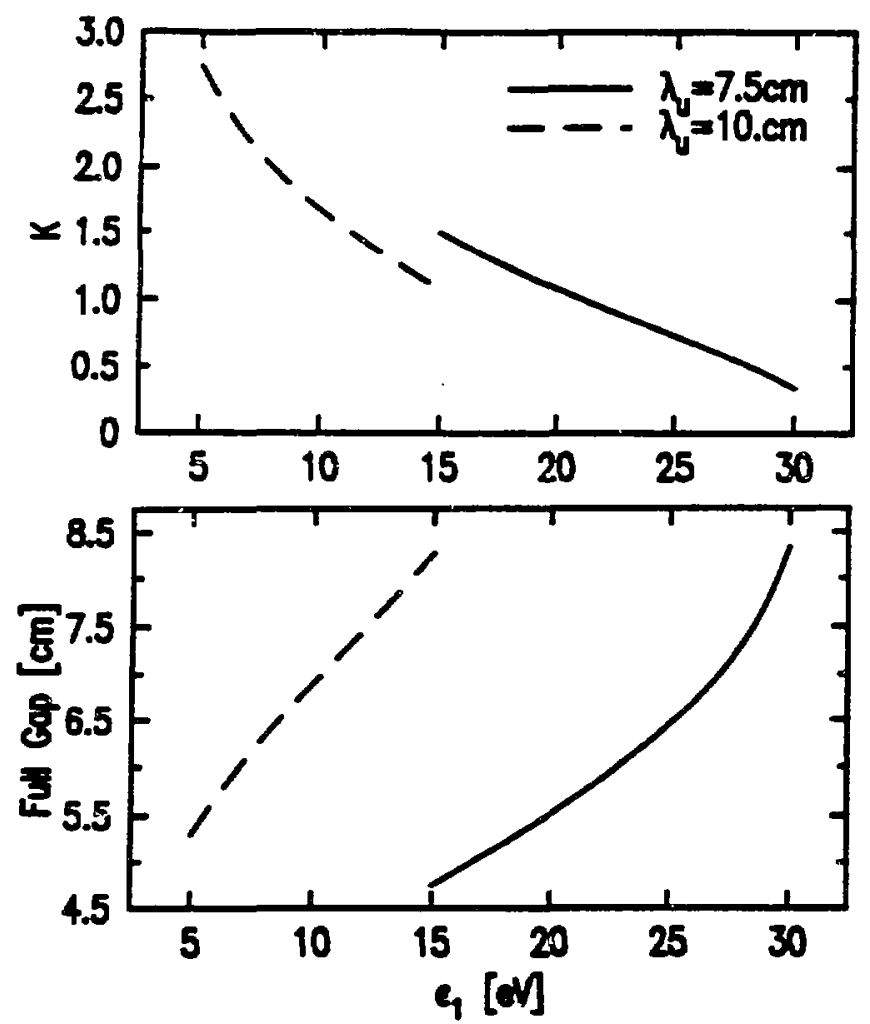

Figure 1: Undulator parameter $\mathbf{K}$ and gap vs fundamental photon energy.

the central cone $N_{1}=1.45 \times 10^{15} \mathrm{ph} / \mathrm{sec} / 0.1 \% /$ Amp.

In previous discussion, all parameters are based on the fundamental $(n=1)$. In fact, we can also use the third harmonic to generate the desired photon spectrum. For example, an undulator of $\lambda_{u}=10 \mathrm{~cm}$ can be used to generate $15-30 \mathrm{eV}$ photon radiation in the third harmonic. The corresponding $K$ value in the region $1.65 \sim 2.74$ in which the photon flux is expected to be near peak in the third harmonic.

\section{Storage Ring}

For our photon energy range, the electron storage ring is chosen to operate at $500 \mathrm{MeV}$. To obtain a dispersion free straight section in the undulator region, we use a Chasman-Green structure. The ring consists of 3 superperiods containing 8 meter straights with roum to accommodate two different $\mathbf{7 . 5}$ meter undulators and a shorter undulator in the injection section. The schematic layout of the electron storage ring is shown in Fig. 2. The betatron and dispersion functions for one superperiod of the lattice without undulators are shown in Fig. 3. The expanded Chasman-Green structure (includes defocussing quadrupoles between the bending magnets) provides better value of the dispersion and betatron functions to facilitate chromaticity correction. We list the main ring parameters in Table 2 and 3.

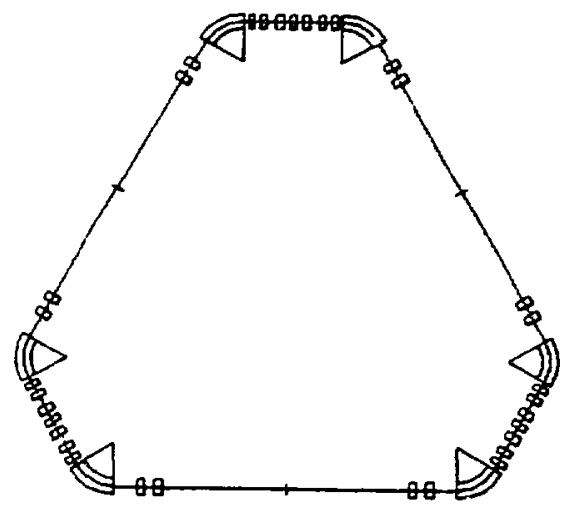

Figure 2: Schematic layout of the electron storage ring with $8 \mathrm{~m}$ straights for undulator radiation.

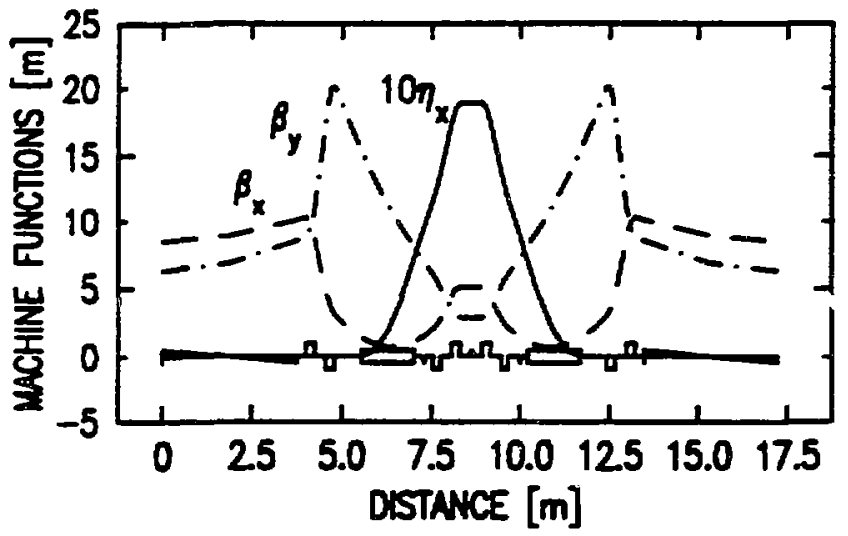

Figure 3: Lattice functions in one superperiod of the ring.

The undulators breair the symmetry of the ring, since they have different periods $\lambda_{u}$ and magnetic parameters $K$. The effects on the ring due to the undulators are investigated at the case in which the maximum designed $K$ values are reachea. After adjusting the strength of the quartrupoles, the distortions of betafunction are confined to the insertions.

\section{Lifetime}

The lifetime of a stored beam is determined from a combination of the gas scattering lifetime and the Touschek lifetime [3]. The total gas scattering lifetime depends on Coulomb scattering and Bremsstrahlung scattering. For our ring, assuming the pressure of the residual diatomic gas in the ring is 1 ntorr with the average atomic number $Z=7$, these lifetimes are 9.70 and 53.88 hours respectively. The total gas scattering lifetime is about 8.22 hours. Touschek lifetime with 1 Amp of beam is computed using ZAP code [4] taking into account bunch lengthening and intrabeam scattering which has small effects. Assuming $Z_{\text {eJ! }}=5 \Omega$, the bunch length is about 3.15 times higher than natural value and Touschek hali-life is 7.06 hours. If $\mathcal{Z}_{\text {ej }}=2 \Omega$, the bunch lengthens about 2.32 times the natural value 
Table 2: Storage Ring Parameters

\begin{tabular}{|l|c|}
\hline Energy, E[MeV] & 500 \\
\hline Superperiod, $N_{,}$ & 3 \\
\hline Circumference, C [m] & 51.635 \\
\hline Lattice Structure & Chasman-Green \\
\hline Dipole Field, $B_{0}[T]$ & 1.2 \\
\hline Bending Radius, $\rho[\mathrm{m}]$ & 1.3903 \\
\hline Horizontal Betatron Tune, $\nu_{x}$ & 3.175 \\
\hline Vertical Betatron Tune, $\nu_{y}$ & 1.125 \\
\hline Uncorrected Chromaticity, $\xi_{x}, \xi_{y}$ & $-3.82,-5.84$ \\
\hline Momentum Compaction, $\alpha$ & $2.927 \times 10^{-2}$ \\
\hline Emittance, $\epsilon$ [m-rad] & $7.309 \times 10^{-8}$ \\
\hline Energy Loss/Turn [KeV] & 3.977 \\
\hline Natural Energy Spread & $3.636 \times 10^{-4}$ \\
\hline Natural Bunch Length [cm] & 3.022 \\
\hline Average Ring Current [mA] & 10.00 \\
\hline Average Current $/$ Bunch $[\mathrm{mA}]$ & 11 \\
\hline Peak Current/Bunch $[\mathrm{A}]$ & 24.2 \\
\hline Coupling Coefficient, $\chi$ & 0.1 \\
\hline Harmonic Number, $b$ & 9 \\
\hline RF Frequency, $[\mathrm{MHz}]$ & 52.254 \\
\hline RF Acceptance, $\epsilon_{R F}[\%]$ & 2.13 \\
\hline
\end{tabular}

and Touschek half-life is 4.55 hours. For the latter, The overall beam half-life from gas and Touschek scattering is 3.29 hours. The lifetime could be increased with the addition of a fourth harmonic cavity to further lengthen the bunch.

\section{Dynamic Aperture}

The natural chromaticities of the ring are corrected to zero by the sextupoles in the achromatic bend which have strengths $S F=12.52 \mathrm{~m}^{-3}$ and $S D=-14.82 \mathrm{~m}^{-3}$. The dynamic aperture of the ring is examined numerically by particle tracking, and is shown in Fig. 4, which is found using the computer code Krackpot [5]. Krackpot which uses an explicit sympletic integrator based on the exact Hamitonian for the drift and isomagnetic combined function bending magnets can track particles without large storage ring approximations. An arbitrary order Taylor map through a succession of elements can be generated by the code through the numerical differential algebra. The resulting geometric aberration coefficients are $\pi_{x x}=-2.47, \pi_{y y}=-119.26$ and $\pi_{x y}=5.24$. Synchrotron oscillations are taken into account in the particle tracking. This aperture is obtained at the center of the insertion and is larger than the assumed physical size of the chamber. Therefore the effects due to the chrornaticity-correcting sextupoles are acceptable.

To find the effects of the undulator on the dynamic aperture, we need to track the electrons with the undulators. This is one of the future works we are going to do. The non-
Table 3: Lattice Parameters

\begin{tabular}{|l|c|c|}
\hline Component & Length $(\mathrm{m})$ & Strength \\
\hline D1 & 4.000 & \\
\hline Q1 & .250 & $3.433\left[\mathrm{~m}^{-2}\right]$ \\
\hline D2 & .300 & \\
\hline Q2 & .250 & $-3.311\left[\mathrm{~m}^{-2}\right]$ \\
\hline D3 & .750 & \\
\hline B & 1.456 & $1.2[T]$ \\
\hline D4 & .150 & \\
\hline SD & .200 & $-14.82\left[\mathrm{~m}^{-3}\right]$ \\
\hline D5 & .200 & \\
\hline Q3 & .200 & $-1.310\left[\mathrm{~m}^{-2}\right]$ \\
\hline D4 & .300 & \\
\hline Q4 & .250 & $2.609\left[\mathrm{~m}^{-2}\right]$ \\
\hline D4 & .200 & \\
\hline SF/2 & .100 & $12.52\left[\mathrm{~m}^{-3}\right]$ \\
\hline
\end{tabular}

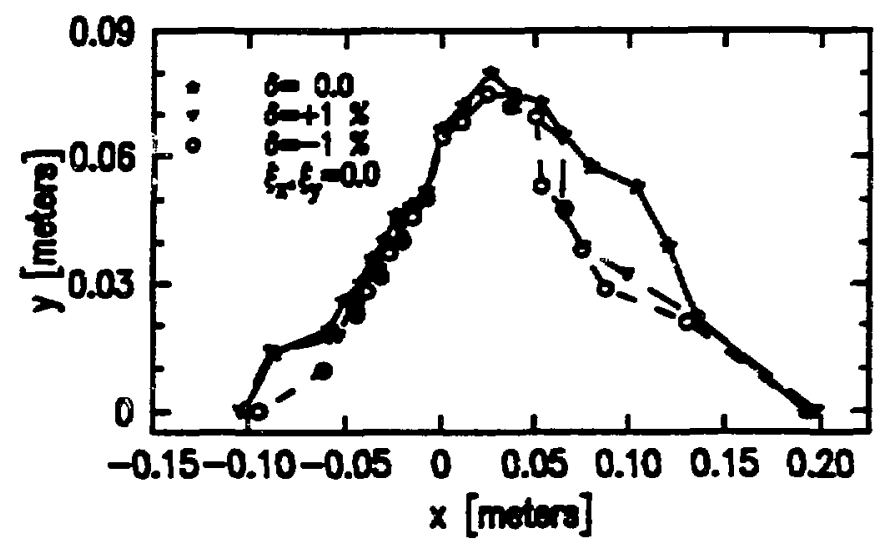

Figure 4: Dynamic aperture from Krackpot 1000 turns.

linear effects due to the sextupoles are expected to not be very important, but the effects due to the undulators have to be investigated. In addition, the breaking of symmetry of the lattice may reduce the aperture. The closed orbit error may also reduce the aperture. For the real aperture, we need to take them all into account.

\section{References}

[1] J. B. Murphy, BNL Report 42333 (1990).

[2] S. Krinsky, IEEE Trans. on Nucl. Sci. NS-30 3078 (1983).

[3] J. Leduff, Nucl. Inst. \& Meth. A239 83 (1985).

[4] M. S. Zisman, S. Chattopadhyay, and J. J. Bisognano, LBL Report 21270 (1986).

[5] M. F. Reusch, E. Forest, and J. B. Murphy, 1991 IEEE Particle Accelerator Conference, San Francisco, May 6-9, 1991. 